\title{
The Cytokine Interleukin-6 Is Sufficient But Not Necessary to Mimic the Peripheral Conditioning Lesion Effect on Axonal Growth
}

\author{
Zixuan Cao, ${ }^{1}$ Ying Gao, ${ }^{1}$ J. Barney Bryson, ${ }^{1}$ Jianwei Hou, ${ }^{1}$ Nagarathnamma Chaudhry, ${ }^{1}$ Mustafa Siddiq, ${ }^{1}$ \\ Jennifer Martinez, ${ }^{1}$ Tim Spencer, ${ }^{1}$ Jason Carmel, ${ }^{2}$ Ronald B. Hart, ${ }^{2}$ and Marie T. Filbin ${ }^{1}$ \\ ${ }^{1}$ Department of Biological Sciences, Hunter College, The City University of New York, New York, New York 10021, and ${ }^{2}$ W. M. Keck Center for Collaborative \\ Neuroscience and Department of Cell Biology and Neuroscience, Rutgers University, Piscataway, New Jersey 08854
}

Lesioning the peripheral branch of a dorsal root ganglion (DRG) neuron before injury of the central branch of the same neuron enables spontaneous regeneration of these spinal axons. This effect is cAMP and transcription dependent. Here, we show that the cytokine interleukin-6 (IL-6) is upregulated in DRG neurons after either a conditioning lesion or treatment with dibutyryl-cAMP. In culture, IL-6 allows neurons to grow in the presence of inhibitors of regeneration present in myelin. Importantly, intrathecal delivery of IL-6 to DRG neurons blocks inhibition by myelin both in vitro and in vivo, effectively mimicking the conditioning lesion. Blocking IL-6 signaling has no effect on the ability of cAMP to overcome myelin inhibitors. Consistent with this, IL-6-deficient mice respond to a conditioning lesion as effectively as wild-type mice. We conclude that IL-6 can mimic both the cAMP effect and the conditioning lesion effect but is not an essential component of either response.

Key words: IL-6; conditioning lesion; regeneration; cAMP; microarray; cytokine

\section{Introduction}

After injury to the adult mammalian CNS, the ensuing failure of axonal regeneration can be attributed to myelin-associated inhibitors of regeneration, the glial scar, and the neuronal intrinsic growth status. Recent attempts to encourage regeneration by adult neurons have centered around elimination of the inhibitory signals via either blocking common receptors (Bradbury et al., 2002; Wang et al., 2002a,b; Domeniconi et al., 2002; Wong et al., 2002) or deletion of the inhibitory molecules themselves (Simonen et al., 2003; Zheng et al., 2003). We and others have begun to elucidate the signaling mechanisms that alter the intrinsic growth state of the neuron such that axons will regenerate in the presence of CNS inhibitors (Filbin, 2003).

Recently, cAMP levels were shown to have an integral role in determining the extent of axonal regeneration in vitro and in vivo. Elevation of neuronal cAMP with neurotrophins or with an analog [dibutyryl-cAMP (db-cAMP)] was shown to overcome in-

Received Feb. 22, 2006; revised April 5, 2006; accepted April 11, 2006.

This work was supported by grants from the New York State Spinal Cord Fund, the National Institutes of Health (NIH) (Grant NS37060), the National Multiple Sclerosis Society, and by core facility grants from the Research Centers for Minorities Institute-NIH and Specialized Neuroscience Research Programs-NIH (Grant NS41073). We thank Marie Handler for outstanding technical support, Dr. Lloyd Williams for help with the image analysis, and Dr. Christine Cain for assistance with this manuscript.

Correspondence should be addressed to Dr. Marie T. Filbin, Department of Biological Sciences, Hunter College The City University of New York, 695 Park Avenue, New York, NY 10021. E-mail: Filbin@genectr.hunter.cuny.edu.

J. B. Bryson's, J. Carmel's, and T. Spencer's present address: Columbia University, 701 West 168th Street, New York, NY 10032.

Z. Cao's and Y. Gao's present address: Wyeth Neuroscience, Wyeth Ayerst Research, Princeton, NJ 08543.

D0I:10.1523/JNEUROSCI.0815-06.2006

Copyright $\odot 2006$ Society for Neuroscience $\quad$ 0270-6474/06/265565-09\$15.00/0 hibition of regeneration by myelin in vitro in a transcriptiondependant manner (Cai et al., 1999; Gao et al., 2004). In addition, evidence suggests that increased endogenous cAMP levels are responsible for the observed spontaneous regeneration of neonatal CNS axons after injury (Cai et al., 2001). Also, in a known situation in which spontaneous regeneration of adult spinal axons does occur, namely that of dorsal column axons subsequent to lesioning of the peripheral branch of the same neurons (Richardson and Issa, 1984; Neumann and Woolf, 1999), application of exogenous cAMP to dorsal root ganglia (DRGs) effectively mimicked the conditioning lesion both in vitro and in vivo (Neumann et al., 2002; Qiu et al., 2002).

Therefore, the question has become what genes are regulated by this increase in neuronal cAMP? Recently, we have shown that de novo synthesis of the enzyme arginase I (Arg I), a key enzyme in polyamine synthesis, is initiated by elevated cAMP, in a cAMP response element-binding protein (CREB)-dependent manner, and that increased polyamine synthesis improves axonal regeneration on an inhibitory substrate (Cai et al., 2002; Gao et al., 2004). Here, we report the identification of another gene that is regulated by both elevating cAMP and by a peripheral conditioning lesion: the cytokine interleukin-6 (IL-6).

In the CNS, IL-6 is found in neurons, glial cells, and macrophages (Schobitz et al., 1992; Gadient and Otten, 1995), and, although expression levels are low under normal conditions, increases occur after brain injury, inflammation, and neuronal disease (Strauss et al., 1992; Tarkowski et al., 1995). Proposed roles for IL-6 include glia proliferation, neuronal survival and differentiation, and pro-inflammatory activities (Hama et al., 1991; 
Hirota et al., 1996; Klein et al., 1997; Marz et al., 1997; Loddick et al., 1998). Now we show that this pleiotrophic cytokine can also promote axonal regeneration in an inhibitory environment, both in vitro and in vivo. In addition, IL-6 is sufficient, but not necessary, to mimic cAMP and the conditioning lesion effects on regeneration. We also show that this effect is dependent on interaction with a known signaling pathway.

\section{Materials and Methods}

RNA preparation. DRG neurons from postnatal day 5 (P5) rat pups were isolated, and $3 \times 10^{4}$ neurons were incubated for $18 \mathrm{~h}$ at $37^{\circ} \mathrm{C}$ in the presence or absence of $1.5 \mathrm{~mm}$ db-cAMP. The cells were then collected and resuspended in TRIZOL solution (Invitrogen, San Diego, CA). The samples were extracted with chloroform, and the aqueous phase was adjusted to $35 \%$ ethanol and loaded onto an RNeasy (Qiagen, Chatsworth, CA) column. RNA was then purified following the manufacturer's protocol. Levels of IL-6 mRNA were normalized to those of glyceraldehyde-3-phosphate dehydrogenase.

RNA was also prepared from DRGs after a peripheral lesion as follows. P21-P23 rats were anesthetized by isoflurane inhalation, and a unilateral sciatic nerve transection was performed at mid-thigh. Animals were killed at $18 \mathrm{~h}$ after lesion, and lumbar 4-5 DRGs were isolated and immediately frozen on dry ice. The ganglia were then homogenized in TRIZOL solution, and RNA was extracted using the RNeasy RNA isolation kit (Qiagen) as per the manufacturer's instructions.

Microarrays. The design, construction, and hybridization of microarrays has been described previously (Pan et al., 2002; Carmel et al., 2004). Briefly, 65-70 nucleotide oligonucleotide probes were obtained from Sigma-Genosys (Woodlands, TX) or custom synthesized. Our arrays also include "alien" oligo controls (Stratagene, La Jolla, CA), for which we have matching synthetic mRNA "spikes" to provide internal standardization (Dudley et al., 2002). Microarrays were printed on poly-L-lysinecoated glass slides using an OmniGrid microarrayer (GeneMachines, San Carlos, CA) and quill-type printing pins (Telechem, Sunnyvale, CA). RNA was prepared as described above. Hybridization probes are prepared using the Genisphere (Montvale, NJ) 3DNA dendrimer system. Automated hybridizations were performed using a Ventana Discovery System (Ventana Medical Systems, Tuscon, AZ). After removal from the instrument, arrays were washed, spin-dried in a centrifuge, and scanned on a GenePix 4000B (Molecular Devices, Union City, CA). Data were exported to GeneSpring (Agilent, Palo Alto, CA) for analysis.

Quantitative real-time PCR. Microarray results were confirmed by comparison with mRNA levels obtained by quantitative reverse transcription-PCR (Q-RT-PCR) using selected gene-specific primer pairs. RNA was reverse transcribed with SuperScript II and random primers as suggested by the manufacturer. The PCRs were performed using $10 \mathrm{ng}$ of cDNA, $50 \mathrm{~nm}$ of each primer, and SYBR Green master mix (Applied Biosystems, Piscataway, NJ) in $10 \mu$ reactions. Levels of Q-RTPCR product were measured using SYBR Green fluorescence (Ririe et al., 1997; Wittwer et al., 1997) collected during real-time PCR on an Applied Biosystems 7900HT system. A control cDNA dilution series was created for each gene to establish a standard curve. Each reaction was subjected to melting-point analysis to confirm single amplified products.

Western blot analysis and immunoassay for IL-6. DRG and hippocampal neurons were isolated from P5 and P1 rat pups, respectively, as described previously (Mukhopadhyay et al., 1994; De Bellard et al., 1996). A total of $1 \times 10^{6}$ cells were plated onto each of the poly-L-lysine-coated wells in 24 -well tissue culture plates and incubated for $24 \mathrm{~h}$ at $37^{\circ} \mathrm{C}$. The supernatant was collected, and IL-6 levels were measured immediately either via a competitive immunoassay kit (R \& D Systems, Minneapolis, $\mathrm{MN}$ ) according to the manufacturer's instructions or via Western blot analysis as follows. Twenty milligrams of total protein were loaded and separated by $10 \%$ SDS-PAGE. After electrophoresis, the separated protein was transferred onto polyvinylidene difluoride membrane (Millipore, Bedford, MA) and probed with an antibody to IL-6 (Santa Cruz Biotechnology, Santa Cruz, CA) overnight at $4^{\circ} \mathrm{C}$. Blots were then further incubated with an anti-animal IgG-HRP antibody (1:5000) for $1 \mathrm{~h}$, and detection was performed using the ECL (Amersham Biosciences) chemi- luminescence system. Each condition was repeated in quadruplicate for at least three separate experiments.

Electrophoretic mobility shift assays. Synthetic oligonucleotides containing the CRE binding site (5'-TAAATGACGTCACAT- 3 ') derived from the IL-6 promoter as well as an oligomer representing the consensus CRE sequence ( $5^{\prime}$-GCCTGACGTCGTCAGAG-3') were annealed and $5^{\prime}$ end-labeled with ${ }^{32} \mathrm{P}$ (PerkinElmer, Wellesley, MA) using T4 polynucleotide kinase (Promega, Madison, WI). Binding reactions contained 50,000-100,000 cpm of labeled probe, various amounts of phosphorylated recombinant CREB (Active Motif, Carlsbad, CA) [phosphorylated by protein kinase $\mathrm{A}$ (PKA) using an in vitro kinase assay (Upstate, Charlottesville, VA)] in binding buffer (20 mм HEPES, pH 7.9, $25 \mathrm{~mm} \mathrm{KCl}, 0.1$ mм EDTA, 5\% glycerol, 2 mm $\mathrm{MgCl}_{2}, 0.5 \mathrm{~mm} \mathrm{DTT}, 0.025 \% \mathrm{NP}-40$, and $0.1 \mathrm{mg} / \mathrm{ml} \mathrm{BSA}$ ) in a total volume of $20 \mu \mathrm{l}$, and a 20 -fold mass increase poly $(\mathrm{dI} \cdot \mathrm{dC})$ over probe. For competition assays, 25-, 50-, and 100-fold excess of unlabeled, annealed oligomer as well as unlabeled, mutated CRE sequence ( $5^{\prime}$-ATATAAGGCCTCATA-3') from IL-6 promoter were included with corresponding labeled probe. Mixtures were then incubated for $30 \mathrm{~min}$ at room temperature, loaded onto a $6 \%$ native polyacrylamide gel, and electrophoresed in $0.5 \times$ Tris-borate EDTA buffer at $100 \mathrm{~V}$ for $2 \mathrm{~h}$. Gels were then dried and exposed to x-ray film.

Intrathecal delivery of IL-6 in rats before neurite outgrowth assay. P21P23 rats (Long-Evans, female) were anesthetized by inhalation of isoflurane. Before implantation, Alzet (Cupertino, CA) 1002 osmotic minipumps were filled with either $0-20 \mathrm{ng} / \mathrm{ml}$ recombinant IL-6 (R \& D Systems) or saline, attached to a cannula, and incubated at $37^{\circ} \mathrm{C}$ for at least $4 \mathrm{~h}$ before implantation. During implantation, the lumbar spinal cord was exposed by laminectomy between L5 and L6. A prefilled cannula was inserted under the dura membrane along the dorsal surface of the cord until the tip rested between L4 and L5. Either IL-6 or saline was delivered at a flow rate of $0.25 \mu \mathrm{l} / \mathrm{h}$. Twenty-four hours after initiation of treatment, the animals were killed, and L4 and L5 DRGs were isolated and dissociated.

Neurite outgrowth assay. The neurite outgrowth assay on MAGexpressing Chinese hamster ovary $(\mathrm{CHO})$ cells or myelin was performed as described previously (Mukhopadhyay et al., 1994; Cai et al., 1999). Briefly, $5 \times 10^{4}$ isolated P1 hippocampal neurons, P5 DRG neurons, or P21-P23 rats after intrathecal delivery of IL-6 were plated onto confluent monolayers of control and MAG-expressing CHO cells or purified myelin $(2 \mu \mathrm{g} / \mathrm{well})$ in eight-chamber tissue culture slides (Lab-tek, Christchurch, New Zealand). Where indicated, KT5720 (200 nм; Calbiochem, La Jolla, CA), 5,6-dichloro-1- $\beta$-D-ribofuranosylbenzimidazole (DRB) ( $5 \mu \mathrm{M}$; Sigma, St. Louis, MO), db-cAMP (1 mM; Calbiochem), or recombinant IL-6 (10-400 ng/ml; R \& D Systems) were added to the cultures. The cocultures were incubated for $18 \mathrm{~h}$ at $37^{\circ} \mathrm{C}$, fixed for $30 \mathrm{~min}$ with $4 \%$ paraformaldehyde, permeabilized with ice-cold methanol, and immunostained with a rabbit polyclonal antibody against GAP43 (1: 4000; from R. Curtis and G. Wilkins, Imperial College, London, UK). The slides were coverslipped in Permafluor mounting medium (Immunon) and viewed under a fluorescence microscope. The length of the longest neurite for each GAP43-positive neuron for the first 180-200 neurons encountered when scanning the slide in a systematic manner was determined using the Simple PCI image analysis program.

Dorsal column lesion. Eight-week-old female Long-Evans rats $(n=17)$ were anesthetized by isoflurane inhalation, and a bilateral dorsal column lesion (DCL) was performed at the T6-T7 level, as described previously (Qui et al., 2002). Intrathecal delivery of IL-6 $(n=7)$ or vehicle solution $(n=5)$ was via a prefilled catheter, attached to an Alzet 1002 minipump, inserted through the atlanta-occipital membrane so that the tip was immediately rostral to the DCL. Intrathecal treatment persisted for 2 weeks, after which the pump was removed and checked for residual volume (the catheter was left in place to prevent further damage to the spinal cord), and the animals were left for an additional 4 weeks before retrograde tracing of the dorsal column axons with HRP applied via the left sciatic nerve. All animals were overdosed with a ketamine/xylazine mixture and perfused transcardially with a solution of $2 \%$ paraformaldehyde and $1.5 \%$ glutaraldehyde in phosphate buffer $5 \mathrm{~d}$ after the tracer injection. Five control animals also received a conditioning lesion of the left sciatic nerve $7 \mathrm{~d}$ before DCL and were allowed 6 weeks to recover, before being 
traced as described above. Fixed tissue was then frozen in OCT embedding medium (Electron Microscopy Sciences, Hatfield, PA), and $30 \mu \mathrm{m}$ sections were cut on a cryostat (Leica, Nussloch, Germany). Sections were stained for HRP (using tetramethylbenzadine) and visualized by dark-field light microscopy. Axonal regeneration was measured as the maximum fiber extension relative to the epicenter of the lesion site.

Conditioning lesion in IL-6-deficient mice. Eight-week-old male mice, wild type $(n=15)$ and IL-6-I- $(n=21)$ (The Jackson Laboratory), were used for this study. Both wild-type and IL-/ - mice were genotyped by genomic PCR analysis, using standard techniques. For conditioning lesion, mice were anesthetized, and the right sciatic nerve was exposed at the mid-thigh level and transected unilaterally. After $7 \mathrm{~d}$, animals were re-anesthetized and L4 and L5 DRGs were isolated and used for in vitro neurite outgrowth assays, or animals were subjected to DCL for in vivo regeneration studies. Briefly, laminectomies were performed at the midthoracic vertebra to expose the spinal cord. The dorsal cord midline was identified and incised to a depth of $1 \mathrm{~mm}$. In some animals, DCL alone was performed and used as controls. After 6 weeks, the regenerating dorsal column fibers were labeled using a transganglionic retrograde tracer, biotinylated cholera toxin $\beta$ subunit (CTb) (List Biologic, Campbell, CA). Animals were re-anesthetized, the sciatic nerve was re-exposed, and $1 \mu \mathrm{l}$ of $\mathrm{CTb}$ was injected into the right sciatic nerve at a site rostral to the peripheral lesion using a 32 gauge Hamilton needle.

Three days after CTb injection, animals were perfused as described above using $4 \%$ cold paraformaldehyde, thoracic spinal cords were dissected out, and sagittal sections at $30 \mu \mathrm{m}$ were cut through the lesion site. The sections (floating) were immunolabeled using goat anti-CTb (1: 10,000; List Biologic) and Vectastain Elite ABC reagents (Vector Laboratories, Burlingame, CA). The sections were developed using hydrogen peroxide $(0.003 \%)$ and diaminobenzidine $(0.02 \%)$. Axonal regeneration was assessed by quantifying the $\mathrm{CTb}$-immunolabeled fibers into and beyond the lesion site using MetaMorph image analysis software (Universal Imaging, Downington, PA). Digital images of a series of three sagittal sections showing maximum axonal growth per animal were chosen. Blocks of $100 \times 100 \mu \mathrm{m}^{2}$ areas were drawn at distances $0-0.6$, $0.6-1.2,2.4-3.6$, and $3.6-4.8 \mathrm{~mm}$ rostral and $0-0.6 \mathrm{~mm}$ caudal to the lesion along the dorsal column. We then set the optical density threshold in each image and quantified the pixel area within each block. Data are presented as mean \pm SEM axonal area $\left(\mu \mathrm{m}^{2}\right)$.

\section{Results}

IL-6 is upregulated after either a peripheral conditional lesion or treatment of neurons with db-cAMP

Previously, we and others showed that the ability of db-cAMP and of a peripheral conditioning lesion to block inhibition by MAG and myelin were each transcription dependent (Smith and Skene, 1997; Cai et al., 2002). Furthermore, we also showed that Arg I was upregulated and was required to overcome inhibition in response to either treatment. To identify other genes that may be involved in the ability of neurons to grow on MAG and myelin, we performed a microarray analysis with mRNA from DRG neurons $18 \mathrm{~h}$ after treatment with db-cAMP $(1 \mathrm{mM})$ or $18 \mathrm{~h}$ after a conditioning peripheral lesion. The microarray was custom made and consisted of 65-70-mer oligonucleotide sequences found in known genes. A comparison of genes that changed more than twofold in response to db-cAMP with a one-sided Student's $t$ test $p$ value of $<0.05$ revealed that 11 increased and 4 decreased. Of the 11 genes that increased, the increase in mRNA for IL- 6 was the greatest $(14.3 \pm 0.7$-fold). Other mRNAs that increased included neuropeptide Y, CREM (cAMP response element modulator), VGF (nerve growth factor-inducible growth factor), and Arg I. The observation that Arg I also increased under both of these conditions is a good positive control, indicating that the microarray analysis is a true representation of genes that have increased under these conditions. However, to confirm that the increases we observe are real, we performed Q-RT-PCR on
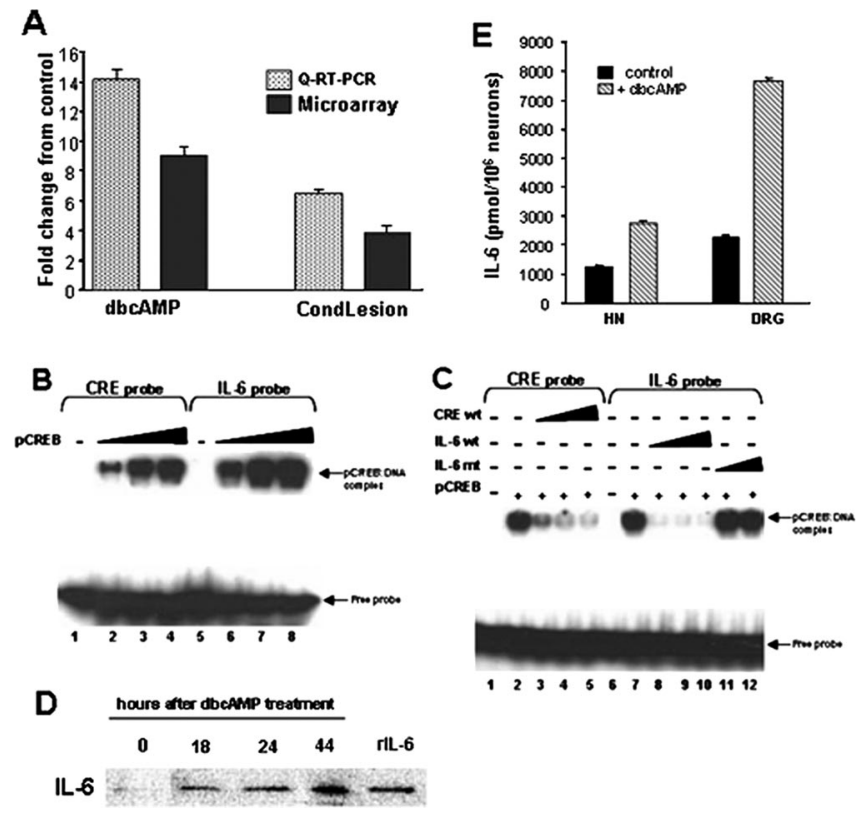

Figure 1. IL-6 increases in response to either elevated CAMP or a conditioning lesion. $\boldsymbol{A}$, Dissociated DRG cells were either treated in culture for $18 \mathrm{~h}$ with $1 \mathrm{~mm} \mathrm{db}$-CAMP or removed from the animal $18 \mathrm{~h}$ after a peripheral lesion (CondLesion), and mRNA was isolated and subjected to either microarray analysis or Q-RT-PCR for IL-6. The control was mRNA from untreated, unlesioned animals. Each experiment was performed at least three times. $\boldsymbol{B}$, Radiolabeled consensus CRE (lanes 1-4) and IL-6 CRE (lanes 5-8) probes were incubated with $25 \mathrm{ng}$ (lanes 2 and 6), $50 \mathrm{ng}$ (lanes 3 and 7), and $100 \mathrm{ng}$ (lanes 4 and 8) of PKA-phosphorylated recombinant CREB. C, Unlabeled, annealed oligomers representing the CRE consensus (CRE wt) and IL-6 CRE (IL-6 wt) sequences were added in 50-fold (lanes 3 and 8), 100-fold (lanes 4 and 9), and 200-fold (lanes 5 and 10) excess over labeled CRE consensus or IL-6 (RE probes to compete for binding of $50 \mathrm{ng}$ of PKA-phosphorylated recombinant CREB (lanes 2-5 and 7-12). A mutated IL-6 CRE sequence (IL-6 mt) was also used to compete for binding of protein at 50 -fold (lane 11) and 100-fold (lane 12) excess over labeled probe. D, DRG cells were cultured with $1 \mathrm{~mm} \mathrm{db-CAMP}$ for the times indicated, after which the cells were lysed and subjected to immunoblotting for IL-6. Recombinant IL-6 (rlL-6) was used as a positive control. E, Hippocampal neurons (HN) or DRG neurons were cultured with $(\mathbb{Q})$ or without $(\square) 1 \mathrm{~mm} \mathrm{db}$-cAMP for $24 \mathrm{~h}$, after which time the supernatants were collected and subjected to an ELISA for IL-6. Results are expressed as the picomole of IL-6 secreted from $10^{6}$ cells in that time. Error bars indicate SE of at least three experiments.

mRNA from DRG neurons treated with db-cAMP or after a conditioning lesion. Figure $1 A$ shows that by RT-PCR, similar increases in IL- 6 mRNA are observed to those seen using microarrary analysis after db-cAMP treatment and are also substantially increased after a conditioning lesion.

Previously, we reported that the cAMP effect on regeneration is dependent on activation of the transcription factor CREB (Gao et al., 2004). Now we show that an IL-6 CRE sequence in the promoter region of the gene binds to phosphorylated CREB (pCREB). Figure $1 B$ shows that when radiolabeled IL-6 CRE is incubated with pCREB, a complex forms that migrates more slowly through the gel compared with unbound probe. The binding of pCREB to IL-6 CRE is concentration dependent because as the amount of pCREB increases, so does the amount of complex formed. In addition, the binding of radiolabeled IL- 6 CRE is specific, because competition with unlabeled IL-6 CRE diminishes the amount of complex, whereas a mutant IL-6 CRE has no effect (Fig. 1C). As a positive control, we show binding of pCREB to an oligomer containing a consensus CRE sequence. These observations are consistent with our working model that cAMP overcomes inhibition in a CREB-dependent manner.

To ensure that, as well as the mRNA, IL-6 protein secretion is 

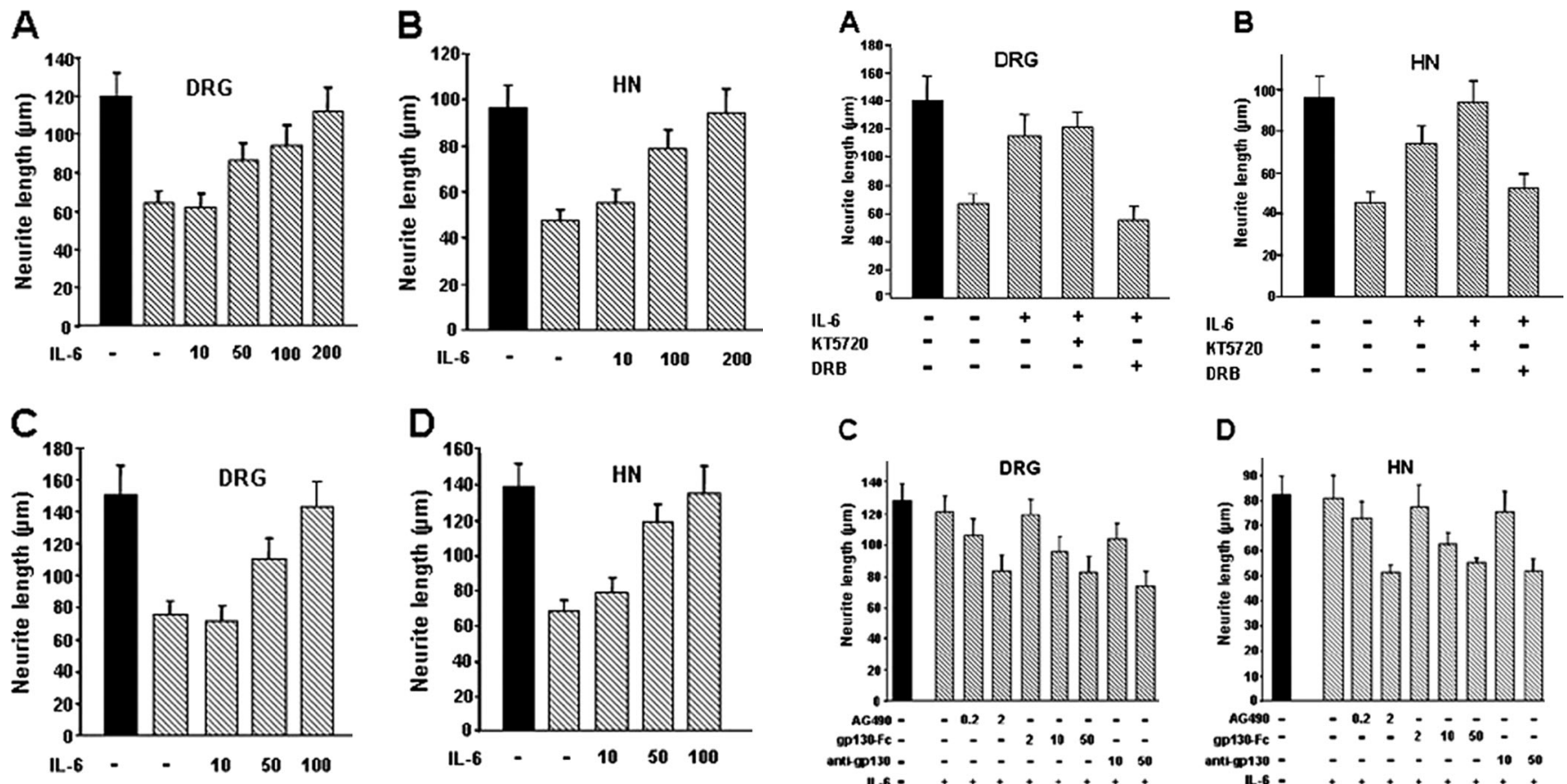

Figure 2. Exogenous IL-6 overcomes inhibition by MAG and myelin in a dose-dependent manner. Isolated P5 rat pup DRG neurons $(A, C)$ or hippocampal neurons (HN; $B, D)$ were dissociated and cultured on MAG-expressing $\mathrm{CHO}$ cells $(\mathbb{\mathbb { Q }} ; \boldsymbol{A}, \boldsymbol{B})$, on control $\mathrm{CHO}$ cells $(\boldsymbol{\square} ; \boldsymbol{A}, \boldsymbol{B})$, or on a substrate of myelin $(\mathbb{\mathbb { N }} ; \boldsymbol{C}, \boldsymbol{D})$ or poly-L-lysine $(\square ; C, D)$ in the presence or absence of various concentrations of IL-6 overnight before being fixed and stained for $\beta$-III tubulin. Results represent the average length of the longest neurite from 180 to 200 neurons \pm SEM from at least three experiments.

also increased, dissociated DRG cells were treated for various times with db-cAMP. The supernatant from the cultures was collected and assessed for the presence of IL- 6 either by Western blotting or by ELISA. As can be seen from the Western blot immunostained for IL-6, secretion of the protein increases with time and is highest at the latest time point, $44 \mathrm{~h}$ (Fig. 1D). This suggests that the neurons are continually expressing IL-6 in the time examined and that it is accumulating in the media. Recombinant IL- 6 was used as a positive control. Similar results were obtained using an ELISA for IL-6 in that the concentration of IL-6 increased in the media of both DRG and hippocampal neurons after db-cAMP treatment. The increase, however, was greater for DRG neurons (Fig. $1 E$ ). Together, these results show that expression of IL-6 is increased under two conditions, elevation of cAMP and after a conditioning lesion, each of which allow neurons to extend neurites on MAG and myelin.

\section{IL-6 overcomes inhibition of neurite outgrowth by MAG and CNS myelin}

To determine whether IL-6 could indeed overcome inhibition by MAG and myelin, as we had shown was the case for db-cAMP, DRG or hippocampal neurons were cultured, in the presence of various concentrations of IL-6, on MAG-expressing CHO cells or purified CNS myelin as a substrate. As can be seen in Figure 2, IL-6 overcomes inhibition by MAG and myelin in a dosedependent manner. Inhibition by MAG is completely blocked by IL- 6 at a concentration of $200 \mathrm{ng} / \mathrm{ml}$ (Fig. $2 A, B$ ), and the neurite length on myelin with IL-6 is the same as from neurons grown on poly-L-lysine (Fig. 2C,D). Even at the highest concentrations used, IL-6 did not have any obvious effects on toxicity or cell death. Although these are mixed cultures, the effect of IL-6 is likely to be a direct effect on the neurons rather than an indirect

C

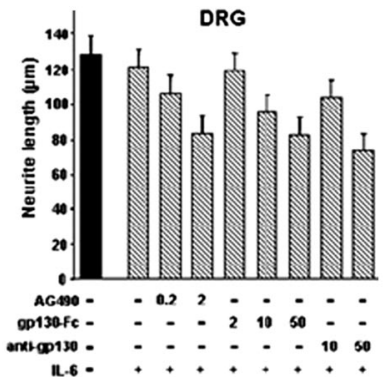

D

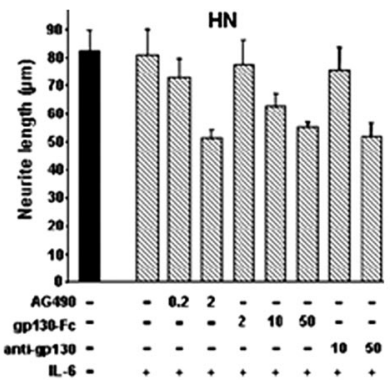

Figure 3. IL-6 overcomes inhibition in a transcription-dependent, cAMP-independent manner via the IL-6R/gp130 receptor complex. DRG neurons ( $\boldsymbol{A}, \boldsymbol{C})$ or hippocampal neurons (HN; $\boldsymbol{B}$, D) were plated onto either MAG-expressing $(\mathbb{Q})$ or control CHO cells $(\square)$ in the presence or absence of IL-6 (20 ng/ml), a PKA inhibitor (KT5720, $200 \mathrm{~nm})$, an inhibitor of transcription (DRB, $5 \mu \mathrm{M}$ ), a JAK inhibitor (AG490, 0.2 or $2 \mu \mathrm{M}$ ), a soluble form of gp130 (gp130-Fc; 2, 10, or 50 $\mu \mathrm{g} / \mathrm{ml}$ ), or an antibody to gp130 (anti-gp130, 10 or $50 \mu \mathrm{g} / \mathrm{ml}$ ) as indicated and cultured overnight before being fixed and immunostained for $\beta$-III tubulin. Results represent the average length of the longest neurite from 180 to 200 neurons \pm SEM from at least three experiments.

effect via glia. This is indicated by the observation that cerebellar neurons, which express only very low levels of the IL-6 receptor (IL-6R), do not respond to IL-6 in our neurite outgrowth assay (data not shown).

The ability of IL- 6 to overcome inhibition by MAG and myelin is transcription dependent, cAMP independent, and via the IL-6R/gp130 receptor complex

To begin to understand what mechanisms are involved in the ability of IL- 6 to overcome inhibition, we first asked whether there was further activation of the cAMP/PKA pathway. To do this, an inhibitor of PKA, KT5720, was included in the cultures along with IL-6. KT5720, at a concentration that blocks the dbcAMP effect, had no effect on the ability of IL-6 to overcome inhibition of neurite outgrowth by MAG for both DRG and hippocampal neurons (Fig. $3 A, B$, respectively). It is possible that IL-6 induces another round of transcription to bring about its effects. To determine whether this is the case, the transcription inhibitor DRB was included in the cultures. Figure 3, $A$ and $B$, shows that for both DRG and hippocampal neurons, the ability of IL-6 to overcome inhibition by MAG is blocked by DRB and therefore is transcription dependent. DRB had no effect on the ability of neurons to extend neurites when grown on control cells, not expression MAG (data not shown). This shows that the DRB effect on the IL-6 ability to block inhibition is not a consequence of a general block of growth.

IL-6 has been shown to affect many cellular processes such as transcription and differentiation by a signaling cascade that is initiated by binding to its receptor, the IL-6R, and its coreceptor, 
A

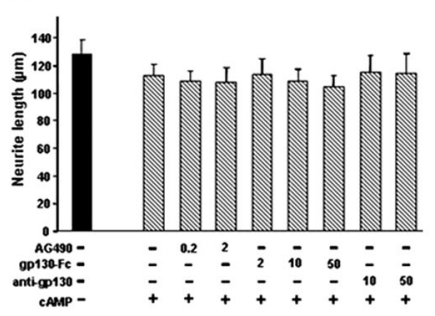

B

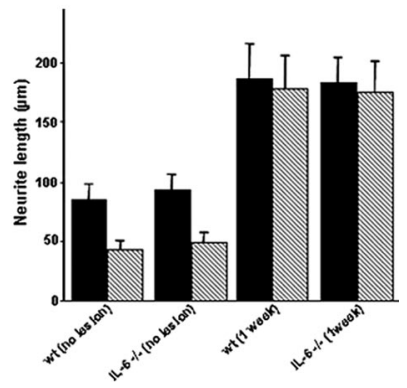

Figure 4. IL-6 is not necessary for db-cAMP or a conditioning lesion to overcome inhibition. DRG neurons from unlesioned animals $(\boldsymbol{A})$ or from animals 1 week after a conditioning peripheral lesion $(\boldsymbol{B})$ were plated onto either MAG-expressing $(\mathbb{Q})$ or control $(\mathbf{\square})$ cells and cultured overnight with or without db-cAMP (1 mM) in the presence or absence of AG490 $(0.2$ or $2 \mu \mathrm{M})$, gp130-Fc (2, 10, or $50 \mu \mathrm{g} / \mathrm{ml})$, or an antibody to gp130 (10 or $50 \mu \mathrm{g} / \mathrm{ml})$ as indicated. The cultures were fixed and stained for $\beta$-III tubulin. Results represent the average length of the longest neurite from 180 to 200 neurons \pm SEM from at least three experiments.

gp130, which in turn activates the Janus-activated kinase (JAK) family of tyrosine kinases (Heinrich et al., 1998). To first determine whether this same receptor complex and signaling pathway is responsible for IL-6 overcoming inhibition of neurite outgrowth by MAG/myelin, we used the following: (1) an antibody to block IL-6 binding to gp-130; (2) a soluble, chimeric form of gp130 consisting of the extracellular domain of gp130 fused to Fc, which functions as a dominant negative to compete with gp-130 for ligand binding; or (3) an inhibitor of JAK, AG490. Figure 3 shows that in a dose-dependent manner, the addition of any one of these compounds blocks the ability of IL-6 to overcome inhibition of neurite outgrowth by MAG for both DRG (Fig. 3C) and hippocampal (Fig. 3D) neurons. These results provide strong evidence that IL-6 is indeed binding to the IL-6R/gp130 receptor complex and signaling through JAK to overcome inhibition by MAG.

\section{IL-6 is sufficient to overcome inhibition but not necessary for the db-cAMP or conditioning lesion effects}

Because we have shown that IL-6 is upregulated by cAMP and can overcome inhibition by MAG/myelin, the next question is whether activation of this IL-6R/gp130 receptor complex and signaling through JAK is necessary for db-cAMP to overcome inhibition. We found that neither gp130 antibody or gp130-Fc nor inhibition of JAK with AG490 had any effect on the ability of db-cAMP to overcome inhibition by MAG (Fig. 4A). These results indicate that although IL-6 is upregulated in response to $\mathrm{db}$-cAMP and is sufficient to overcome inhibition, it is not necessary for the db-cAMP-induced blockade of MAG-mediated inhibition.

To test this possibility further, we investigated the ability of DRG neurons from IL-6-deficient mice (IL-6-/-) (Kopf et al., 1994) to grow on MAG after a conditioning lesion. Our hypothesis is that the conditioning lesion effect is brought about by a transient increase in cAMP, which leads to an increase in IL-6. If IL-6 is indeed sufficient but not necessary for cAMP to overcome inhibition, as suggested by the results in Figure $4 A$, then the absence of IL-6 should have no effect on the ability of a conditioning lesion to allow DRG neurons to grow on MAG or myelin. This is indeed the case. At 1 week after peripheral lesion, DRGs from either wild-type or IL-6-/- mice were able to extend long processes on MAG-expressing cells (Fig. 4B). Therefore, in the
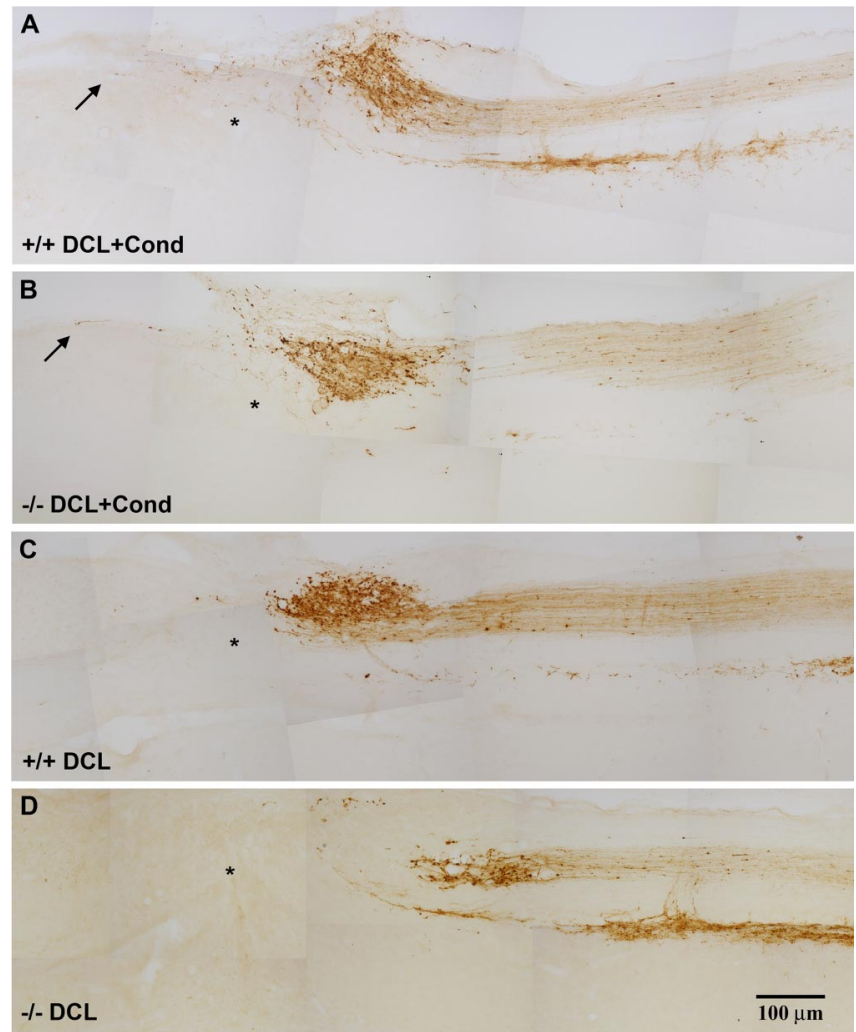

Figure 5. IL-6 is not necessary for the conditioning lesion effect on dorsal column regeneration. Representative sagittal sections of the dorsal spinal cord showing (Tb-immunolabeled axons through the lesion site are shown. Many regenerating axons attempting to grow through and beyond the lesion site were observed in both wild-type and IL- $6-/-$ mice ( $\boldsymbol{A}, \boldsymbol{B}$, arrows). Without a previous conditioning lesion, most injured axons retracted from the injury site and formed dystrophic ends in both wild-type $(\boldsymbol{C})$ and IL-6-/ $-(D)$ mice. Asterisks indicate the lesion site in the dorsal cord.

absence of IL-6, a conditioning lesion still allows DRG neurons to overcome inhibition by MAG.

The effect of a conditioning lesion on subsequent dorsal column regeneration was then compared between the wild-type and IL-6-/ - mice. Initially, when we used our established method of transganglionic labeling of dorsal column axons by injecting tracer into the sciatic nerve 4 weeks after the peripheral lesion, we saw no labeled axons in or even near the DCL site. In animals that received only DCL or nonlesioned controls, the tracer transport was normal. Because it has been reported that there is retarded peripheral nerve regeneration in IL-6-/- mice (Zhong et al., 1999), we thought perhaps in these mice, at the time we inject the tracer, the peripheral nerves may not have regenerated sufficiently to take up the tracer. To determine whether this was the issue, the tracer was injected into the sciatic nerve at 6 weeks rather than 4 weeks after peripheral lesion. This resulted in good labeling of dorsal column axons, suggesting that our prediction was correct and the lack of labeling was a consequence of the slowed peripheral nerve regeneration in IL-6-/- mice. A comparison of the extent of axonal regeneration after a conditioning lesion revealed no difference between the IL-6-/ - and wild-type mice; the IL-6-/ - mice responded to a conditioning lesion as well as wild-type mice (Figs. 5, 6). It is of note, however, that the conditioning lesion effect on dorsal column regeneration is less robust in mice than in rats. Others also find a less robust conditioning lesion effect in mice compared with previously published results in rats (C. Ho and M. Tessier-Lavigne, personal communication). 


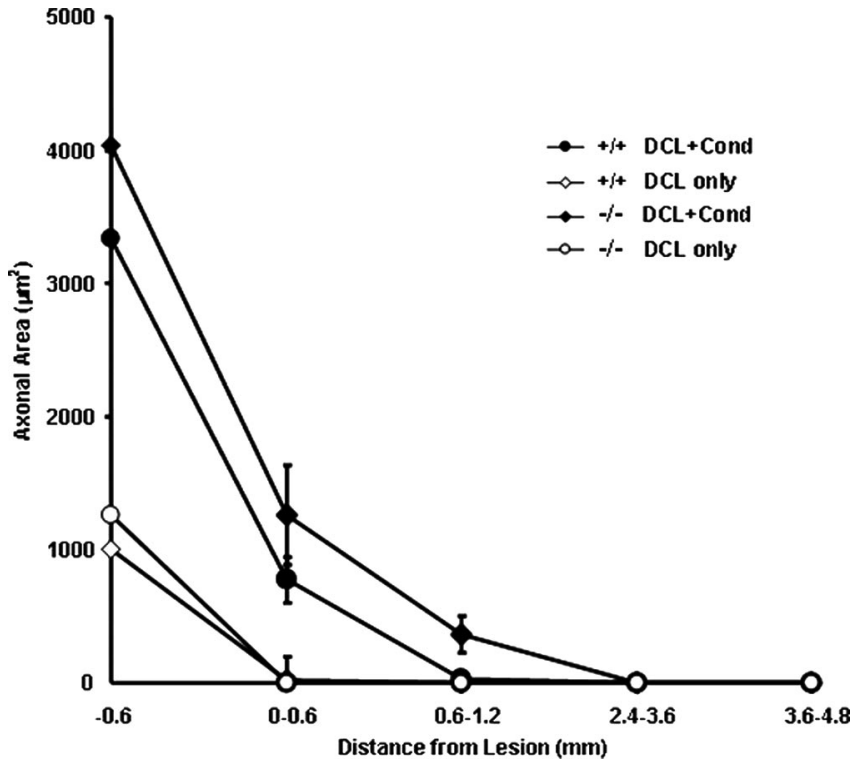

Figure 6. Quantitation of axonal regeneration in IL-6-deficient and wild-type mice. The extent of axonal regeneration was assessed by quantifying the CTb-immunolabeled fibers along the path of axon growth in the dorsal column using MetaMorph image analysis software. There was no significant difference in the extent of regeneration of the injured dorsal column axons between wild-type and IL-6-/ - mice after a peripheral conditioning (Cond) lesion.

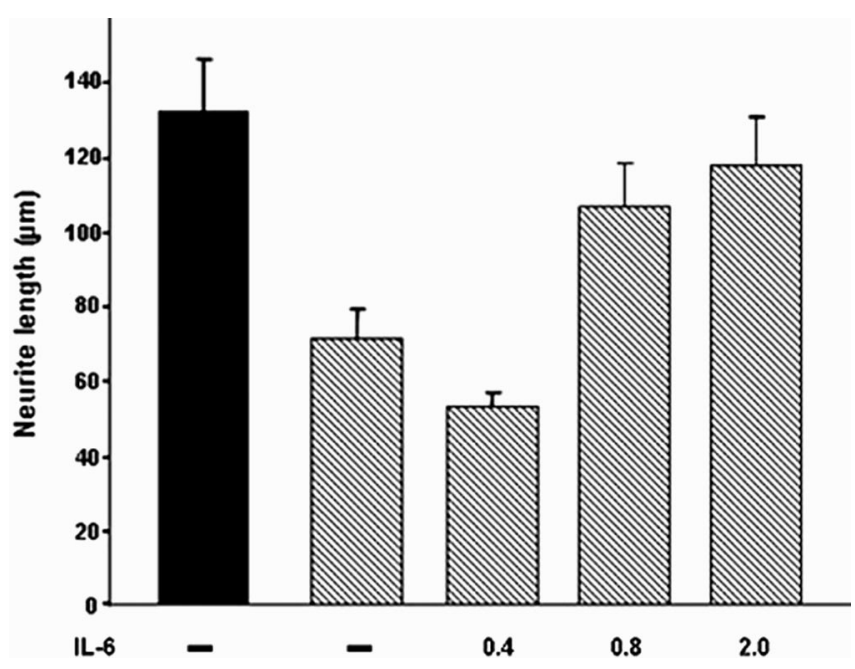

Figure 7. Intrathecal delivery of IL-6 overcomes inhibition by MAG when DRG neurons are subsequently cultured. IL-6, at the doses indicated, was delivered to $\mathrm{P} 60$ rats for $24 \mathrm{~h}$ before the DRG neurons were removed and cultured on either MAG-expressing $(\mathbb{Q})$ or control $(\square)$ CHO cells for $24 \mathrm{~h}$ before being fixed and stained for $\beta$-III tubulin. Results represent the average length of the longest neurite from 180 to 200 neurons \pm SEM from at least three animals under each condition.

\section{Delivery of IL-6 intrathecally blocks inhibition of DRG neurons by MAG in culture}

As an initial assessment of whether IL-6 would be effective in encouraging regeneration in vivo, we delivered recombinant rat IL-6 intrathecally via osmotic mini-pumps, at various doses, for $24 \mathrm{~h}$. DRG neurons from these animals were then removed and cultured to determine whether their neurite outgrowth was still inhibited by MAG. Figure 7 shows that DRG neurons from animals that received IL- 6 intrathecally are not inhibited by MAG. The IL- 6 effect is dose dependent, and a complete block of inhibition by MAG is observed with neurons from animals treated
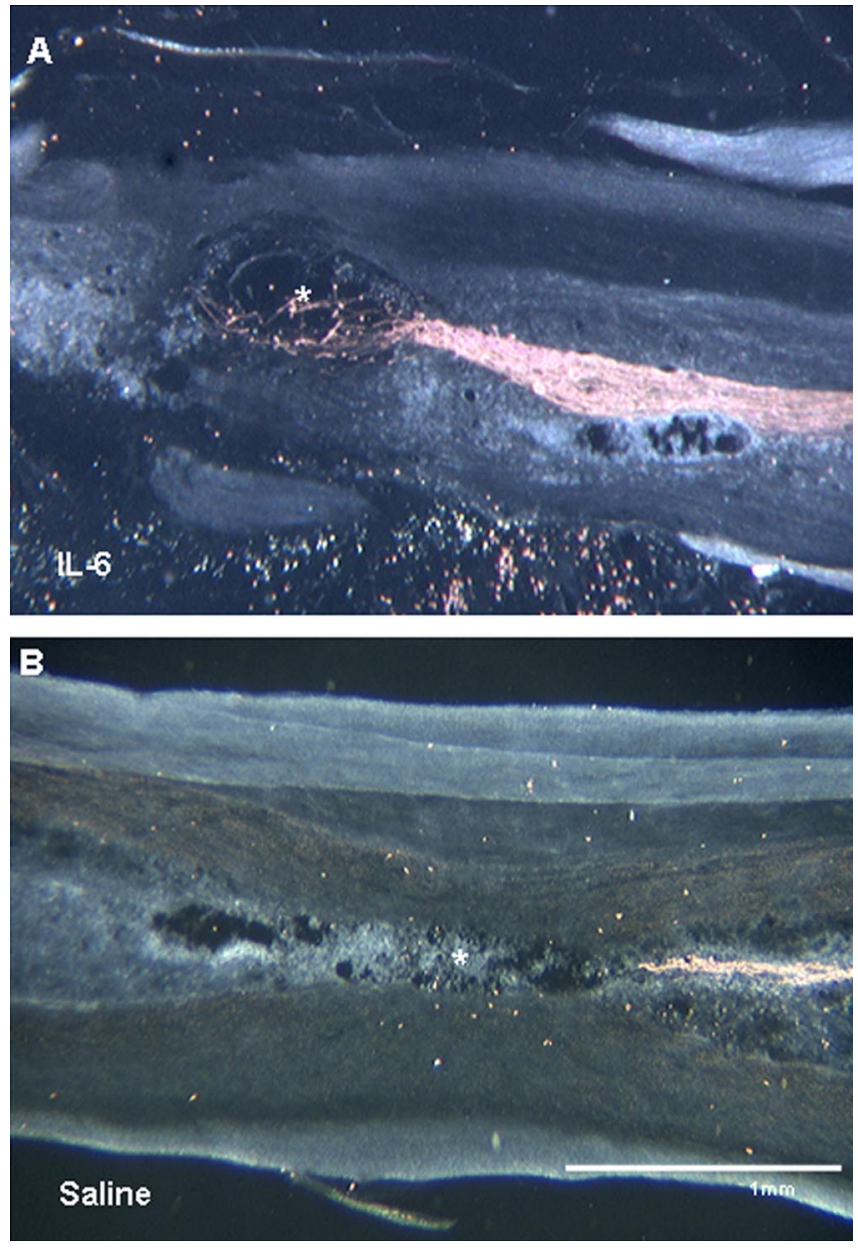

Figure 8. Intrathecal delivery of IL-6 is sufficient to promote dorsal column axon regeneration in vivo. A DCL was performed on $\mathrm{P} 60$ rats at $\mathrm{T} 6 / 7$ and, at the same time, intrathecal minipumps were inserted delivering IL- 6 at a dose of $2 \mathrm{pmol} / \mathrm{kg} / \mathrm{h}(\boldsymbol{A})$ or saline $(\boldsymbol{B})$ for 2 weeks. After an additional 4 weeks, the animals were perfused and sectioned. Five days before the animals were killed, dorsal column axons were retrogradely traced with HRP. The images are longitudinal sections of thoracic spinal cord. The asterisk indicates the lesion center.

with IL-6 at a dose of $2 \mathrm{pmol} / \mathrm{kg} / \mathrm{h}$. Therefore, IL-6 delivered intrathecally reaches DRG neurons and allows them to grow on MAG when subsequently cultured.

\section{Delivery of IL-6 intrathecally promotes regeneration of dorsal spinal axons}

To assess whether IL-6 is sufficient to encourage spinal axonal regeneration in vivo, a bilateral DCL was performed at T6/7 in P21-P23 rats. At the same time as the animals were lesioned, an intrathecal cannula, attached to an osmotic mini-pump, was inserted to continuously deliver IL-6 for 2 weeks at a concentration of $2 \mathrm{pmol} / \mathrm{kg} / \mathrm{h}$, the optimal dosage as determined by the experiment described in Figure 7. As a control, saline was delivered intrathecally for the same length of time. After 2 weeks, the minipumps were removed and the animals were killed 4 weeks later. Five days before the animals were killed, the dorsal column axons were backfilled with HRP for tracing. Figure $8 B$ shows immunostaining of a typical control animal and, as has been reported numerous times before, there are no regenerated fibers in the lesion site. As is typically observed, the tracer-filled lesioned axons have retracted from the lesion site. In sharp contrast, in the IL-6-treated animals, there are numerous fibers coursing into the lesion site. In some instances, at the tip of the regenerating axons, 
there are what appear to be flattened growth cones (Fig. 8A). When the distance the axons regenerated was measured, for the majority of animals treated with IL-6, the axons regenerated closer to the epicenter of the lesion than for the control animals $(p<0.03)$, and for a subset of IL-6-treated animals, axons passed the lesion epicenter, which was never observed in the control animals. This shows that IL-6 has a general improvement in regeneration compared with control animals. In addition, delivery of IL- 6 to the animals for 2 weeks at $2 \mathrm{pmol} / \mathrm{kg} / \mathrm{h}$ did not appear to have any overt detrimental effects on the animals' behavior.

\section{Discussion}

IL-6 has been reported to have both detrimental and beneficial effects in the nervous system (Gadient and Otten, 1997). Although the detrimental effects are usually attributed to its proinflammatory actions of this cytokine and inflammation after spinal cord injury is traditionally regarded as being detrimental, we show here that exogenous IL-6 is beneficial for axonal regeneration: it not only overcomes myelin inhibitors of regeneration in culture but, when delivered intrathecally, promotes spinal axon regeneration in vivo.

These results are important because, first, exogenous IL-6 can allow neurons to extend neurites in the presence of myelin inhibitors. Second, although not necessary, IL-6 can mimic the effects of both a conditioning lesion and elevation of cAMP in culture and in vivo. Third, exogenous IL-6, delivered intrathecally, is sufficient to promote regeneration of central dorsal column axons. These findings are consistent with our working model in which we propose that the peripheral conditioning lesion effect on regeneration of subsequently lesioned dorsal column axons is brought about by an increase in cAMP, followed by transcription of specific genes (Filbin, 2003). Now we add to this model by including IL- 6 as one of the genes that is upregulated in response to either CAMP or a conditioning lesion. However, although we show that IL-6 is sufficient to mimic the cAMP-dependent, conditioning lesion effect, we suggest that it is not necessary. We reach this conclusion based on two observations. First, agents that block the IL- 6 effect (IL-6 antibodies or gp130-Fc) have no effect on the ability of db-cAMP to overcome inhibitors of regeneration in myelin. Second, we find no difference between DRG neurons from wild-type and IL-6-/- mice in their ability to grow in an inhibitory environment either in culture or in vivo after a conditioning lesion. This sufficiency of, but lack of necessity for, IL-6 in the conditioning lesion/cAMP effect indicates that more than one signaling pathway is activated simultaneously by these treatments and that there is redundancy in these pathways in their ability to overcome myelin inhibitors and encourage regeneration in vivo. Indeed, we have shown that upregulation of the enzyme Arg I and the subsequent increased synthesis of polyamines is also a consequence of the conditioning lesion/cAMP effect (J. Qiu, K. Deng, D. Cai, W. Mellado, and M. T. Filbin, submitted for publication). However, because inhibiting synthesis of polyamines blocks both the cAMP and the conditioning lesion effect, we suggest that, unlike IL-6, upregulation of Arg I is necessary as well as sufficient for this effect.

Although in the studies reported here we found no difference in the IL-6 knock-out and wild-type mice in their response to a conditioning lesion, others have reported impairment in the response of the IL-6-deficient mice (Cafferty et al., 2004). In that study, impairment in the general enhanced neurite outgrowth after a conditioning lesion was reported. Here, we see no difference in the response of IL-6-deficient and wild-type neurons in culture to a conditioning lesion in that there is not only a general improvement in growth on a permissive substrate but also that neither neuron genotype is inhibited by MAG. Consistent with this, we also report no difference in the ability of dorsal column axons of IL-6-deficient and wild-type mice to regenerate when lesioned $7 \mathrm{~d}$ subsequent to a peripheral lesion. In contrast, Cafferty et al. (2004) reported that even after a conditioning lesion in the IL-6 knock-out animals, no dorsal column axons regenerated to reach or cross the lesion site; they behave as if no conditioning lesion had been performed. In contrast, in wild-type mice, they report a conditioning lesion effect (distance regenerated) on dorsal column regeneration equivalent to what we observed in both IL-6-deficient and wild-type mice. We have no explanation for these differences in findings because, to our knowledge, the same strain of mouse was used in both studies (Kopf et al., 1994) (stock number 002254; The Jackson Laboratory). Also, the difference in results does not seem to result from axonal tracing problems because an abundance of dye-filled axons are obvious in all mice in both studies. Furthermore, because the distance regenerated we report for the IL-6 knock-out and wild-type mice is very similar to that observed by Cafferty et al. (2004) for the wild-type mice, it is unlikely that differences in surgical techniques can account for the differences observed in the knock-out mice. Our results, however, are consistent with our observation in rat neurons, namely that IL- 6 is sufficient but not necessary to mimic the cAMP effect. Consistent with the suggestion that parallel pathways are activated, inhibition of the polyamine pathway has no effect on the ability of IL-6 to block inhibition (data not shown). However, cross talk between the polyamine and IL-6 pathways is not yet fully understood.

The immune response and subsequent inflammation in the nervous system in response to injury or disease has been suggested to be "a double-edged sword" (Wyss-Coray and Mucke, 2002). Undoubtedly, after spinal cord injury, the secondary damage caused by inflammation considerably exacerbates the deficits resulting from the injury per se (Schwab and Bartholdi, 1996). On the other hand, a controlled immune response has been reported to be neuroprotective after optic nerve or spinal cord injury (Rapalino et al., 1998; Lazarov-Spiegler et al., 1999). In addition, a recent study shows that if reactive astrocytes are ablated after injury, the ensuing damage after spinal cord injury is much more pronounced (Faulkner et al., 2004). In a similar manner, IL- 6 has been reported to have contrasting effects in the CNS. With time, in transgenic mice in which IL- 6 is expressed under the control of the GFAP promoter, there is considerable spontaneous neurodegeneration (Campbell et al., 1993). In sharp contrast, in these same mice after injury, which results in a dramatic increase in IL-6 in the transgenics relative to the increase in wildtype mice, there is increased wound healing and apparent neuroprotection after trauma (Penkowa et al., 2003a,b). These studies suggest that perhaps the timing and the levels of IL-6 expression dictate whether there is a detrimental or beneficial effect. In the uninjured transgenic, there is persistent expression of IL- 6 that is slightly above control levels; however, after injury, IL-6 levels increase sharply and transiently, as opposed to wild-type animals that exhibit a moderate, persistent increase. Consistent with the hypothesis that slightly elevated levels of IL-6 are detrimental, one study has shown that, after spinal cord injury, blockade of endogenous IL-6, with an antibody to the IL-6R, induced a decrease in GFAP staining and a slight improvement in functional recovery (Okada et al., 2004).

In another study, when fibroblasts genetically engineered to deliver hyper-IL-6 (IL- 6 fused to its $\alpha$ receptor) were grafted into the lesion site, there was no improvement in regeneration (Lac- 
roix et al., 2002). There are at least two possible reasons why no beneficial effects of IL- 6 were seen under these conditions. First, hyper-IL- 6 activates all cells that express gp130 regardless of coreceptor expression, and this could result in the activation of cells that would not react to IL- 6 itself. Second, we show here that when delivered intrathecally, IL-6 must reach the DRG cell bodies because when these neurons are subsequently removed and cultured, they grow in an inhibitory environment. Therefore, it is likely that IL-6 delivered intrathecally is promoting dorsal column regeneration by affecting the neuronal cell body as well as perhaps having a direct effect at the lesion site. In the study in which hyper-IL-6 is delivered via cells grafted at the lesion site, it is very unlikely that IL- 6 would diffuse far enough to reach the cell body.

Here, we also begin to address the mechanism of action of IL-6 in overcoming myelin inhibitors. We have established that it is through activation of the classic IL-6 trimeric receptor, resulting in activation of the JAK signaling cascade. Importantly, we have also shown that the IL-6 effect is transcription dependent. It now remains to be determined which genes upregulated by IL- 6 are responsible for overcoming inhibition by myelin and promoting regeneration in vivo. In addition, it will be of interest to assess whether different genes are upregulated in response to high and low concentrations of IL-6.

In summary, we have shown that IL-6 is upregulated in response to both elevated cAMP and a conditioning lesion and that this increase is sufficient to overcome the myelin-associated inhibitors as well as promote spinal axon regeneration in vivo. However, we also show that although IL-6 is sufficient to overcome inhibition, it is not necessary for cAMP or a conditioning lesion to do so. This raises the possibility that a combination of IL-6 with other treatments that we have shown to be effective in encouraging axonal regeneration, such as elevation of Arg I and polyamine synthesis, may be synergistic and result in greater growth than any treatment alone.

\section{References}

Bradbury EJ, Moon LD, Popat RJ, King VR, Bennett GS, Patel PN, Fawcett JW, McMahon SB (2002) Chondroitinase ABC promotes functional recovery after spinal cord injury. Nature 416:636-640.

Cafferty WB, Gardiner NJ, Das P, Qiu J, McMahon SB, Thompson SW (2004) Conditioning injury-induced spinal axon regeneration fails in interleukin-6 knock-out mice. J Neurosci 24:4432-4443.

Cai D, Shen Y, De Bellard M, Tang S, Filbin MT (1999) Prior exposure to neurotrophins blocks inhibition of axonal regeneration by MAG and myelin via a cAMP-dependent mechanism. Neuron 22:89-101.

Cai D, Qiu J, Cao Z, McAtee M, Bregman BS, Filbin MT (2001) Neuronal cAMP controls the developmental loss in ability of axons to regenerate. J Neurosci 21:4731-4739.

Cai D, Deng K, Mellado W, Lee J, Ratan RR, Filbin MT (2002) Arginase I and polyamines act downstream from cyclic AMP in overcoming inhibition of axonal growth MAG and myelin in vitro. Neuron 35:711-719.

Campbell IL, Abraham CR, Masliah E, Kemper P, Inglis JD, Oldstone MB, Mucke L (1993) Neurologic disease induced in transgenic mice by cerebral overexpression of interleukin 6. Proc Natl Acad Sci USA 90:10061-10065.

Carmel JB, Kakinohana O, Mestril R, Young W, Marsala M, Hart RP (2004) Mediators of ischemic preconditioning identified by microarray analysis of rat spinal cord. Exp Neurol 185:81-96.

De Bellard ME, Tang S, Mukhopadhyay G, Shen YJ, Filbin MT (1996) Myelin-associated glycoprotein inhibits axonal regeneration from a variety of neurons via interaction with a sialoglycoprotein. Mol Cell Neurosci 7:89-101.

Domeniconi M, Cao Z, Spencer T, Sivasankaran R, Wang RC, Nikulina E, Kimura N, Cai H, Deng K, Gao Y, He Z, Filbin MT (2002) Myelinassociated glycoprotein interacts with the Nogo66 receptor to inhibit neurite outgrowth. Neuron 35:283-290.
Dudley AM, Aach J, Steffen MA, Church GM (2002) Measuring absolute expression with microarrays with a calibrated reference sample and an extended signal intensity range. Proc Natl Acad Sci USA 99:7554-7559.

Faulkner JR, Herrmann JE, Woo MJ, Tansey KE, Doan NB, Sofroniew MV (2004) Reactive astrocytes protect tissue and preserve function after spinal cord injury. J Neurosci 24:2143-2155.

Filbin MT (2003) Myelin-associated inhibitors of axonal regeneration in the adult mammalian CNS. Nat Rev Neurosci 4:703-713.

Gadient RA, Otten U (1995) Interleukin-6 and interleukin-6 receptor mRNA expression in rat central nervous system. Ann NY Acad Sci 762:403-406.

Gadient RA, Otten UH (1997) Interleukin-6 (IL-6)—a molecule with both beneficial and destructive potentials. Prog Neurobiol 52:379-390.

Gao Y, Deng K, Nikulina E, Mellado W, Spencer T, Barco A, Kandel E, Filbin MT (2004) Activation of CREB is necessary and sufficient to overcome mylein inhibitors and encourge spinal axon regneration in vivo. Neuron 44:609-619.

Hama T, Kushima Y, Miyamoto M, Kubota M, Takei N, Hatanaka H (1991) Interleukin-6 improves the survival of mesencephalic catecholaminergic and septal cholinergic neurons from postnatal, two-week-old rats in cultures. Neuroscience 40:445-452.

Heinrich PC, Behrmann I, Muller-Newen G, Schaper F, Graeve L (1998) Interleukin-6-type cytokine signalling through the gp130/Jak/STAT pathway. Biochem J 334:297-314.

Klein MA, Moller JC, Jones LL, Bluethmann H, Kreutzberg GW, Raivich G (1997) Impaired neuroglial activation in interleukin-6 deficient mice. Glia 19:227-233.

Kopf M, Baumann H, Freer G, Freudenberg M, Lamers M, Kishimoto T, Zinkernagel R, Bluethmann H, Kohler G (1994) Impaired immune and acute-phase responses in interleukin-6-deficient mice. Nature 368:339-342.

Lacroix S, Chang L, Rose-John S, Tuszynski MH (2002) Delivery of hyperinterleukin-6 to the injured spinal cord increases neutrophil and macrophage infiltration and inhibits axonal growth. J Comp Neurol 454:213-228.

Lazarov-Spiegler O, Solomon AS, Schwartz M (1999) Link between optic nerve regrowth failure and macrophage stimulation in mammals. Vision Res 39:169-175.

Loddick SA, Turnbull AV, Rothwell NJ (1998) Cerebral interleukin-6 is neuroprotective during permanent focal cerebral ischemia in the rat. J Cereb Blood Flow Metab 18:176-179.

Marz P, Herget T, Lang E, Otten U, Rose-John S (1997) Activation of gp130 by IL-6/soluble IL-6 receptor induces neuronal differentiation. Eur J Neurosci 9:2765-2773.

Mukhopadhyay G, Doherty P, Walsh FS, Crocker PR, Filbin MT (1994) A novel role for myelin-associated glycoprotein as an inhibitor of axonal regeneration. Neuron 13:757-767.

Neumann S, WoolfCJ (1999) Regeneration of dorsal column fibers into and beyond the lesion site following adult spinal cord injury. Neuron 23:83-91.

Neumann S, Bradke F, Tessier-Lavigne M, Basbaum AI (2002) Regeneration of sensory axons within the injured spinal cord induced by intraganglionic cAMP elevation. Neuron 34:885-893.

Okada S, Nakamura M, Mikami Y, Shimazaki T, Mihara M, Ohsugi Y, Iwamoto Y, Yoshizaki K, Kishimoto T, Toyama Y, Okano H (2004) Blockade of interleukin- 6 receptor suppresses reactive astrogliosis and ameliorates functional recovery in experimental spinal cord injury. J Neurosci Res 76:265-276.

Pan JZ, Ni L, Sodhi A, Aguanno A, Young W, Hart RP (2002) Cytokine activity contributes to induction of inflammatory cytokine mRNAs in spinal cord following contusion. J Neurosci Res 68:315-322.

Penkowa M, Giralt M, Lago N, Camats J, Carrasco J, Hernandez J, Molinero A, Campbell IL, Hidalgo J (2003a) Astrocyte-targeted expression of IL-6 protects the CNS against a focal brain injury. Exp Neurol 181:130-148.

Penkowa M, Camats J, Hadberg H, Quintana A, Rojas S, Giralt M, Molinero A, Campbell IL, Hidalgo J (2003b) Astrocyte-targeted expression of interleukin-6 protects the central nervous system during neuroglial degeneration induced by 6-aminonicotinamide. J Neurosci Res 73:481-496.

Qiu J, Cai D, Dai H, McAtee M, Hoffman PN, Bregman BS, Filbin MT (2002) Spinal axon regeneration induced by elevation of cyclic AMP. Neuron 34:895-903. 
Qiu J, Deng K, Cai D, Mellado W, Filbin MT (2006) The polyamine putrescine promotes spinal axon regneration in vivo. J Neurosci Submitted.

Rapalino O, Lazarov-Spiegler O, Agranov E, Velan GJ, Yoles E, Fraidakis M, Solomon A, Gepstein R, Katz A, Belkin M, Hadani M, Schwartz M (1998) Implantation of stimulated homologous macrophages results in partial recovery of paraplegic rats. Nat Med 4:814-821.

Richardson PM, Issa VM (1984) Peripheral injury enhances central regeneration of primary sensory neurones. Nature 309:791-793.

Ririe KM, Rasmussen RP, Wittwer CT (1997) Product differentiation by analysis of DNA melting curves during the polymerase chain reaction. Anal Biochem 245:154-160.

Schobitz B, Voorhuis DA, De Kloet ER (1992) Localization of interleukin 6 mRNA and interleukin 6 receptor mRNA in rat brain. Neurosci Lett 136:189-192.

Schwab ME, Bartholdi D (1996) Degeneration and regeneration of axons in the lesioned spinal cord. Physiol Rev 76:319-370.

Simonen M, Pedersen V, Weinmann O, Schnell L, Buss A, Ledermann B, Christ F, Sansig G, van der Putten H, Schwab ME (2003) Systemic deletion of the myelin-associated outgrowth inhibitor nogo-a improves regenerative and plastic responses after spinal cord injury. Neuron 38:201-211.

Smith DS, Skene JH (1997) A transcription-dependent switch controls competence of adult neurons for distinct modes of axon growth. J Neurosci 17:646-658.

Strauss S, Bauer J, Ganter U, Jonas U, Berger M, Volk B (1992) Detection of interleukin-6 and alpha 2-macroglobulin immunoreactivity in cortex and hippocampus of Alzheimer's disease patients. Lab Invest 66:223-230.

Tarkowski E, Rosengren L, Blomstrand C, Wikkelso C, Jensen C, Ekholm S, Tarkowski (1995) Early intrathecal production of interleukin-6 predicts the size of brain lesion in stroke. Stroke 26:1393-1398.

Wang KC, Kim JA, Sivasankaran R, Segal R, He Z (2002a) P75 interacts with the Nogo receptor as a co-receptor for Nogo, MAG and OMgp. Nature 420:74-78.

Wang KC, Koprivica V, Kim JA, Sivasankaran R, Guo Y, Neve RL, He Z (2002b) Oligodendrocyte-myelin glycoprotein is a Nogo receptor ligand that inhibits neurite outgrowth. Nature 417:941-944.

Wittwer CT, Herrmann MG, Moss AA, Rasmussen RP (1997) Continuous fluorescence monitoring of rapid cycle DNA amplification. Biotechniques 22:130-131, 134-138.

Wong ST, Henley JR, Kanning KC, Huang KH, Bothwell M, Poo MM (2002) A p75(NTR) and Nogo receptor complex mediates repulsive signaling by myelin-associated glycoprotein. Nat Neurosci 5:1302-1308.

Wyss-Coray T, Mucke L (2002) Inflammation in neurodegenerative disease-a double-edged sword. Neuron 35:419-432.

Zheng B, Ho C, Li S, Keirstead H, Steward O, Tessier-Lavigne M (2003) Lack of enhanced spinal regeneration in nogo-deficient mice. Neuron 38:213-224.

Zhong J, Dietzel ID, Wahle P, Kopf M, Heumann R (1999) Sensory impairments and delayed regeneration of sensory axons in interleukin-6deficient mice. J Neurosci 19:4305-4313. 